\title{
Investigating teacher perceptions of teaching ICT in Wales
}

\author{
Jan Barnes ${ }^{1}$ (D) Steve Kennewell ${ }^{2}$
}

Published online: 16 November 2016

C The Author(s) 2016. This article is published with open access at Springerlink.com

\begin{abstract}
Regardless of what is intended by government curriculum specifications and advised by educational experts, the ICT competencies or skills taught and learned in and out of classrooms can vary considerably. In this paper, we explore how we can investigate the perceptions that individual teachers have of ICT as a subject, and how these and other factors may influence students' learning. We report small-scale multiple case study research which examines ICT teaching as an activity system and identifies contradictions within the teaching of ICT, highlighting issues concerning the object of the curriculum, the roles of the participants and the cultures of schools. We discuss how these contradictions may be resolved and the emerging variations in the teacher's perceptions of ICT as a subject and their practice within the classroom. Finally we discuss how these variations may impact on future suggested changes to the curriculum in Wales for ICT and Computing, particularly how differences in perception and practice between teachers may impact on pupil experience and the development of the subject.
\end{abstract}

Keywords ICT capability - Teacher perceptions · Activity theory · Contradictions · Practice and pedagogy

\section{Introduction}

In England and Wales, the introduction of the National Curriculum in 1989 required all students between the ages of 5 and 16 to follow the same subject-based curriculum which was to be designed by government based committees and approved by the secretary of

Jan Barnes

janine.barnes@uwtsd.ac.uk

Steve Kennewell

skennewell@cardiffmet.ac.uk

1 University of Wales Trinity St David, Townhill Campus, Townhill Road, Swansea SA2 0UT, UK

2 Cardiff Metropolitan University, 7 Barneshall Avenue, Worcester WR5 3EU, UK 
State. Prior to this, there was little formal basis for the learning of Information and Communication Technology (ICT), although ideas set out by an earlier document from the government inspectorate for Education (DES 1985) strongly influenced the initial ICT curriculum, which characterised the intended outcome of learning as 'ICT Capability' (DES/WO 1990), ). The statutory curriculum has been revised on a number of occasions, and since the devolution of Wales in 1998, there have been differences between the curriculum in England and Wales, with the latest revision in Wales being introduced in 2008 (DCELLS 2008). However, what is taught and learned in different schools, and different classrooms in the same school, may vary considerably from what is intended by authors of curriculum specifications. This is especially the case in Wales, which has retained the loose structure that has been characteristic of the ICT curriculum since its inception. The purpose of this paper is to explore the competences or 'skills' which are inherent to ICT Capability as it is taught within the Welsh education system, and to investigate teachers' individual constructs and perceptions of the subject and the methods they use in the classrooms to develop those skills. Variations in these personal constructs of ICT capability and the methods teachers use are likely to have implications for different experiences for pupils in the classroom. Ultimately this may impact on their level of capability in the subject. Furthermore, differences in personal constructs and classroom practice may have implications for the inclusion of aspects of Computer science in the future curriculum as has been proposed.

There have been several attempts to define the subject area by identifying the key competences within ICT capability. The official definition in Wales includes such aspects as "communicating, problem solving"; "transmitting information and interpreting information conveyed by table, diagrams and models" (ACCAC 2008). A number of authors (e.g. Kennewell et al. 2000), suggest that ICT capability is a combination of the routines, techniques, processes and concepts of ICT coupled with the ability to use higher order skills in identifying their appropriate use or application. Kennewell et al. (2000): 1) suggest that.

Information and communications technology refers to the set of tools used to process and communicate information; to be 'ICT capable' is to be competent in controlling the situations in which those tools are applied.

This refers to the use of higher order skills as well as the tools provided by particular applications; ICT involves deciding which tools to use and how to use them to bring about the optimum solution. This is reinforced by government advice in England (DCFS 2002), which states that students should have the ability and confidence to use ICT equipment and software with purpose to support their work. They should also be able to identify situations where the use of ICT would be relevant. To enable this, students should be able to reflect and comment on the use of ICT, and to recognise that ICT affects the way in which people live and work (Gaskell 2003). These ideas are similar to those of ICT literacy, which Markauskaite (2007) suggests is the use of digital technology, communication tools, and/or networks to access, manage, integrate, evaluate and create in order to function in a knowledge society.

In order to understand the nature of ICT and the key competencies within this subject area, however, it is not enough to analyse what is contained in curriculum specifications and schools' schemes of work. We need to ask how these competencies 
are developed in the classroom and, indeed, if they are best learned within a discrete subject or should they be developed across the curriculum. An investigation of this development of competencies thus also needs to consider the perceptions and practices of the teacher involved, and it is that aspect on which this paper will focus.

\section{Theoretical framework for pedagogical research}

There has been little previous research concerning pedagogy within ICT as a subject, and we have sought suitable frameworks to guide this innovative study. Perhaps the best known model for the processes involved in developing teaching and classroom practice is that of Shulman (1987), based on observations and interviews with a large number of teachers. Shulman's Model of Pedagogical Reasoning (1987), is a model of effective teaching, which links a series of different types of knowledge to create effective practice. The model is not a mechanistic process, but rather a concept that underlies pedagogical practice. It comprises a number of categories to evaluate the knowledge base: Content Knowledge; General Pedagogical Knowledge; Curriculum Knowledge; Pedagogical Content Knowledge; Knowledge of learners and their characteristics; Knowledge of educational contexts; Knowledge of educational ends. One of the key points was the link between the content knowledge and the pedagogical knowledge, that the teaching of a subject will be improved if, as well as specialist knowledge of the subject, teachers also have knowledge of how best to present the concepts and develop the skills inherent to that subject (Shulman 1987). Thus pedagogical content knowledge involves an ability to recognise and generate alternative ways of representing the subject matter (Wilson et al. 1987). This knowledge is particular to the subject matter concerned, so that teachers of ICT are likely to use different pedagogical techniques than, for example, those teaching mathematics or one of the humanities subjects.

Whilst Shulman recognised the role of context as one aspect of teacher knowledge, recent studies of teaching have attached more importance to the school and the wider social and political environment. In the study reported in this paper, the work of Engeström (2001) has been examined in order to establish a possible analytical framework for this study. Cultural Historical Activity Theory (CHAT) allows the researcher to pay particular attention not only to the specific object of activity under research, but also the Vygotskian focus of 'mediation and discourse'. CHAT is a developing resource which has the flexibility to adapt to any given activity within the workplace (Daniels et al. 2010).

The proposition of activity theory is that human activity is a socially-situated phenomenon which consists of much more than mere action. It is a theory or framework that situates practice within an environment and examines the process and the purpose of that practice (Daniels et al. 2010). In doing this, it attempts to account for the complexity of real-time activity, investigating factors that influence the activity such as the beliefs and perceptions of those central to the activity (Engeström et al. 1999). Within CHAT, the activity triangle (Fig. 1) represents the relationships and networks within related activity systems, in which any alteration one aspect of a system, whether it be part of the tools, rules, roles, subject, or object of the activity, is likely to affect another part of the system or systems. The analysis works by examining the individual 
elements of the activity system, and looks for contradictions, which are essentially disturbances within the system (Daniels et al. 2010).

The mediating factors displayed in the activity triangle above were applied to the interviews and the classroom activity and were interpreted in the following manner:

Object This element represents the general aim of the activity - what the subject is trying to achieve. In the case of this study, the object is the development of the key competencies inherent to ICT. These are generally referred to as 'skills' by teachers, a term which can cover a wide range of general competencies and tool-specific techniques.

Subject When analysing activity within the classroom, there are at least two different perspectives, with corresponding activity systems taking place; that of the teacher, whose object is likely to be the learning that they wish to take place, and that of the students, who are more likely to be concerned with the successful completion of the task set by the teacher (for example, the production of a movie). For the purpose of this paper, the analysis of the observations will focus on that of the teacher as the subject and it is their object that will lead the activity triangle.

Tools and artefacts These include the formal curriculum documents; interpretations of the curriculum (e.g. lesson plans); software and hardware; pedagogical tools such as task instructions, guidance materials for pupils; language.

Rules and codes of behaviour This may include the attitude of the participants, for example the motivation of the students; how help is sought in carrying out tasks and also any perceptions of the students of appropriate use of ICT which may be different in their everyday lives from what is expected in the classroom.

Community and organisational structures The culture of both the school and the wider community influence this area of the activity. At the time of the study there was a political drive to change the ICT curriculum, a change which was already taking place in England and was under review in Wales. Community and organisational structures

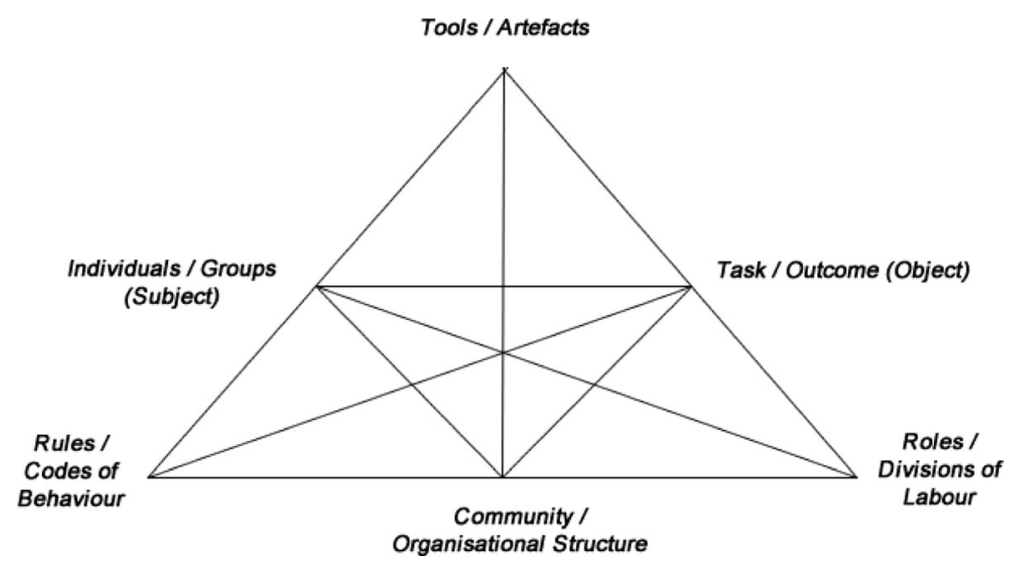

Fig. 1 Activity triangle adapted from Engeström et al. (1999) 
may also include the way that the competencies are perceived, for instance the status of the subject within a community which develops ICT on cross-curricular basis will be less than that of a community developing ICT with discrete lessons.

Roles and divisions of labour The key roles concern the provision of instruction, help and guidance for pupils within the classroom. Support may be provided by teachers, teaching assistants and indeed the pupils themselves if they are actively involved in the learning environment.

This framework does not fully address the detail of classroom interactions which are so important in pedagogical practice, however. Kennewell (2010) suggests the use of a model based upon the analysis of the affordances (or potential for action within the setting) and constraints (the structure allowing that action to take place), which are related to the goals observed within the classroom. Consequently, this model was adopted as a framework for observing and recording classroom practice, details of which can later be extracted for analysis within the activity setting triangle.

\section{Methods}

This research is concerned with why the teachers teach in the way that they do, and how the development of key competencies matches with their own personal constructs. The research also took place at a time of change within the educational system in Wales. Case study is a preferred method when researchers are asking the how and why questions and it is particularly applicable when the researcher has little control over the events taking place in the macro environment (Yin 2009). Consequently, it was decided to use three separate schools as the basis for independent case studies. The three schools represent different ways in which they develop ICT, ranging from a discrete subject environment to an approach where the skills are developed through one week of intense study during the year and supported on a cross-curricular basis. These approaches are indicative of the various strategies employed in welsh schools in the development of ICT capability,

The data was collected within each school over a period of nine months. One teacher in each school undertook an initial interview, a lesson observation and subsequent reflective dialogue, then a repeat of the observation and reflection and finally another interview. The interviews were conducted in order to gain an understanding of the teachers' perception of those skills which are definitive to the subject of ICT, whilst the observations were conducted to establish if the activity within the classrooms supported the development of the skills the teacher had identified. Because each teacher was also looking at their own practice in the development of these skills in their students there was also a post-observation dialogue, in which attempts were made to encourage the teacher to reflect on their own practice prior to a different observation and finally another interview to find out if any of the teachers' earlier perceptions had changed.

Thematic analysis was used for identifying and analysing patterns of meaning in the data and ultimately to highlight the most salient meanings present. The coding has been carried out independently, in that each set of data (interviews, reflective dialogue and observations) has been scanned for themes independently, and then re-scanned in order to establish common themes prior to deeper analysis. The broad themes from Activity 
Theory were used across all the analysis, but the sub-themes that emerged when coding the interview data differed from those found in the observational data.

\section{Results}

The results were analysed in terms of the elements or mediating factors of Engeström's triangle, cross-referenced to the themes and examples emerging from the analysis of classroom observations together with both the initial and final interviews. The three case studies differ in their mode of development of ICT capability and were chosen because of this range of style. Each of the teachers had taught for several years in the same school. For each case, only limited background details concerning the school, the teacher and the observed lesson are provided as the focus in this paper is on how to investigate teacher perceptions and their implications for pedagogy. The results for each school take the form of a brief description of relevant elements of the ICT lesson activity system and the relationships within it. These will be discussed in terms of the contradictions which emerged within and between the case studies.

\subsection{School A}

The pedagogical strategy in school A was to develop ICT capability largely on a crosscurricular basis, with most of the development of pupils' knowledge of software tools occurring over a concentrated week for the entire year group. Each day, the single specialist ICT teacher gave a 'masterclass', which was then supplemented by a team of non-specialist ICT teachers leading 'workshop' lessons. Pupils were taught in groups formed using generic cognitive ability scores assessed by the school. The rationale was that the specialist ICT teacher taught the 'top' set so that they could be 'pushed', whilst the lowest ability set was taught by the non-specialist teacher with the least subject knowledge. The scheme of work and the learning resources were designed by the ICT specialist to differentiate for varying degrees of ability. The differentiation was designed upon the basis of the generic cognitive ability scores and not on the teacher's personal knowledge of the pupils. This scaffolding employed the use of templates and writing frames to assist those considered to have lower ability levels engage with the higher order skills highlighted within the scheme of work. However the pupils were not given adequate tools to engage with this differentiation resulting in a tendency for them to use the support superficially without full activation of any of the higher order thinking skills which the differentiation was aimed to activate.

The analysis highlighted a number of contradictions within School A which warrant further comment. The first contradiction is within the tools/artefacts element. The scheme of work designed by the school supported an opportunity for the pupils to consider and evaluate their work; however, the lesson observations suggested that task sheets which are given to the pupils to help accomplish the task focused only on lower order skills concerning techniques. Furthermore, a second contradiction between the object and the tools/artefacts was evident from the initial interview, where the teacher described the nature of ICT capability as including the use of higher order metacognitive skills, yet the pedagogical tools employed concerned only lower-order techniques. From the analysis of the interview it is clear that the teacher believed that 
ICT capability is about more than the use of techniques within applications, with references to 'problem solving' in the conversation. However, the school culture indicated that the status of ICT was lower in that it was not afforded the same position as other subjects which had at least an hour-long lesson each week and were taught by subject specialists. This contradiction between the community element and the object was reinforced by a parallel contradiction between the object and the roles of the teachers involved. The specialist ICT teacher only taught the 11-14 age group for isolated periods during the school year, so did not know the students and was reliant on general data concerning cognitive ability. Furthermore, the students she was 'pushing' had the ability to explore tools and to orchestrate their own learning, whereas the lower ability pupils needed constraints and affordances to be provided by the teacher, suggesting that those classes with the least cognitive ability would gain most from a specialist teacher with pedagogical content knowledge specific to ICT.

Both classroom observations demonstrated a contradiction between the teaching of low-order techniques observed in lessons and the goals stated in the initial interview which refer to 'problem solving' 'higher order skills' and concepts. However, in the final interview, there was no reference to higher order thinking and the discussion of skills focuses on the techniques of using software tools. At one point the teacher expressed the view that the term 'skills' was synonymous with the term 'tools', indicating that the competencies within ICT were perceived to be of low order. Thus a change in the perception of the competencies was emerging, in that the teacher's perception is moving from one incorporating metacognition to a merely tools-based definition which matches the practice observed. This resolved some of the contradictions but created a new one between the object (learning to use software tools) and the artefacts (the scheme of work and the National Curriculum).

\subsection{School B}

The teacher in School B used a system of facilitation; he presented the pupils with a problem to be solved and informed them of the available help sheets, supportive videos and online tutorials to help them to solve a problem. Subsequently, they were asked to developing tutorials for their peers of what they had learned. This enabled the pupils to take control of their learning, and stimulated reflection on their new knowledge.

The analysis of School B focussed on the teacher's perception of what ICT Capability entails and how it is best developed pedagogically. Throughout the analysis, all mediating factors supported the teacher's claims that ICT capability comprises not just knowledge of the techniques for using individual software applications but also the metacognitive skills needed to successfully decide what tools should be used and to what effect. The development of this capability was supported in a corresponding way, with the role of the teacher being that of a facilitator giving greater control to the pupils. The exception to this arose during the initial interview in that the teacher saw his belief that to effectively develop ICT capability, they need to develop independent learning, problem solving and the ability in the pupils to think for themselves as being in discord with the National Curriculum for Wales (2008). In reality, the National Curriculum orders show a clear link with the need to teach problem solving and thinking skills.

The majority of the contradictions emerged in analysis of the final interview and appear to be a direct result from the changes taking place externally in the political 
arena. These changes relate to the review of the curriculum taking place in Wales and the changes in the curriculum initiated in England which impacted on the perception of the teacher interviewed. Primarily there is a contradiction between the development of the pupil-centred independent learning approach as devised by the ICT department of School B for 11-14 year olds and the teacher-centred approach used by other teachers in the department. One interpretation of this is that the culture of the organisation recognises the high status of ICT and the need to develop metacognitive skills, but in reality there is insufficient confidence amongst the majority of the staff to develop it in this way. This results in a contradiction within the object between the activity settings in different classrooms. Furthermore, the teacher felt that the proposed changes to the curriculum did not go far enough and that there was a need to teach aspects of hardware and computer and network engineering,

\subsection{School C}

Finally the analysis of School C showed that, like School B, there was a high status given to the subject: ICT was timetabled as a specialist subject for an hour per week for 11-14 year olds and the school employed a team of specialist ICT teachers in order to develop ICT capability. They also used a system of 'learning ladders' and peer support through a 'buddy' system as a form of pedagogical tool, enabling the pupils to generate discussion and verbalisation of and about their learning. The learning ladders were constructed by the pupils in the class and consisted of the success criteria which the pupils considered important to enable them in solving the problem and completing the task. The formation of the criteria and the priorities in which they placed the criteria on the 'learning ladder' involved verbalisation of both the problem and the most appropriate way in which it could be solved. Peer support, where pupils in need of help first asked designated team 'buddies' before engaging with the teacher enabled further discussion and in so doing required further use of the pupils higher order thinking skills. The pupils knew that any help provided had to be verbal in nature, rather than taking over the computer. This required the pupils to put their learning into their own words, thus supporting further reflection on their learning. A number of key contradictions emerged in analysing one ICT teacher's perceptions of ICT capability, and establishing whether the classroom practices observed supported their personal constructs. There was a clear perception that ICT capability comprises knowledge of the tools of the application, the associated terminology and ultimately the ability to use the tools and terminology appropriately in the problem solving process. There was also an implication that this is developed in a linear way, with the pupils first mastering the techniques before moving on to the use of terminology and finally developing the capacity to apply the techniques in solving problems. However, obsservations showed that the use of discussion and dialogue was central to the pedagogical strategy from the start. This dialogue took place on two levels: between the teacher and the pupils during question and answer phases of the lesson, and amongst pupils through the use of discussion. There was a need for a shared understanding of the associated terminology and a degree of developing understanding of appropriate use of the software to be in place in order that there was successful use of dialogue in the development of ICT capability. This meant that ICT capability development was more holistic than linear in its structure, and led to a contradiction between the tools and roles in the classroom 
activity system. Regarding the proposed changes to the curriculum, the teacher felt that the curriculum did not require change at all but that those teachers need to be more innovative and motivational in their approach to the development of ICT capability.

\section{Discussion}

The main findings from the teacher case studies concern variation in both perception of ICT capability and in pedagogical practice. The case study teachers were initially in agreement with regard to their perception of ICT capability, in that they included higher order metacognitive skills as part of their explanation of their understanding. This corresponds to the ethos of the national curriculum, (ACCAC 2000, 2008) and indeed the definitions of ICT Capability (Kennewell 2003). The National Curriculum orders clearly refer to the use of 'Thinking Skills' within the teaching of the subject, and Kennewell (2003), p. 7) describe ICT capability as being a non-linear process involving the simultaneous development of "routines, techniques, key concepts, processes and higher order skills". However, further probing reveals a more complex relationship in each school setting, with a number of issues which have been characterised as contradictions. In addition, the variations between schools suggest that teachers' perception of ICT capability may range on a continuum from predominantly loworder skills or techniques to predominantly higher-order skills involving metacognition. There may also be a similar continuum of pedagogical strategy, ranging from a teachercentred, mechanistic approach to a pupil-centred, problem-solving approach.

The analysis of the classroom practice of this teacher, as noted in the results section for School A, highlighted that the approach used to develop ICT capability in the school and the strategies from a teaching perspective were indeed more associated with the development of a tool-based subject. Furthermore, the subject was not held in high status within the school community as it was not afforded regular discrete lessons within the curriculum. So the contradiction seems to have been resolved, but not through professional learning resulting in a change in practice so as to achieve the object, but a recognition that the features of the setting were constraining the practice resulting in a change in the object.

In addition, the school allocated pupils to teaching groups based on a belief in fixed general cognitive ability, so that those pupils who are of a lesser ability have more restrictions and rigidity in the scheme of work and are taught by non-specialists. This reduced the likelihood that the teachers would feel able to introduce higher-order skills in 'lower ability' groups. However, if one believes that the capability being developed, particularly any digital capability, can be directly related to the capability of the teacher (Lankshear 2010; Webb 2002) then one might expect that those groups who were less able and possibly in need of greater support in the development of ICT capability might be associated with the teachers having the greatest ICT capability.

The reasoning given for associating the high ability groups with the more ICTcapable teachers was that the higher ability groups would require greater challenge; however, those pupils are likely to have the metacognitive ability which enables them to create opportunities to construct their own knowledge.

Shulman's (1987) model of pedagogical reasoning suggests that in order to effectively differentiate for the lower ability groups there is a need for teacher to possess a 
knowledge of the subject, a knowledge of how to teach the subject and a knowledge of how the particular pupils learn. The development of a scheme of work and differentiation by a teacher who has limited knowledge of the pupils contradicts Shulman's model.

The contradictions which emerged in Schools B and C were not between their perceptions of ICT capability and their classroom practice. Both teachers defined ICT capability as having a tools-based component, where there was a need to be able to operate a variety of software applications, but also highlighted the need to be able to use higher order metacognitive skills in discussing the subject, to use the language and terminology of the subject, and to use the software applications in an appropriate and effective way to solve problems. Their classroom practice both supported their personal constructs of ICT capability with the development of the higher order metacognitive skills alongside the operational techniques. However, there was a different contradiction within the pedagogical strategies and tools used to develop ICT capability. In school B there was an ethos of facilitation; ICT capability, the metacognitive skills associated with it and the language of the subject were taught in a holistic way, but this was only put into practice by one teacher. School $\mathrm{C}$ adopted a more linear format, with the belief that first you had to teach ICT techniques before developing the language associated with the subject, but expected pupils to understand and use the language to talk about their work from the start.

In school B the teacher felt that the National curriculum did not include the need to develop independent problem solving skills whereas his personal perception included this as a competency within ICT capability. In reality the National curriculum clearly identifies the use of creativity and problem solving as a competency associated with ICT capability and as such there is no contradiction in existence. However, if this is not recognised by the teacher, there is a danger that the perceived contradiction may lead to an unnecessary change in their teaching practice to a more mechanistic approach. Further differences emerged in the way they interpreted the proposed changes the National Curriculum to include computer architecture and programming. The teacher in School B felt that the proposed changes did not go far enough, in contrast to the teacher in School C who felt that it was the teachers' approach which needed to change rather than the curriculum.

The examination of practice in the three case studies suggested that there may be a continuum of approaches to the development of ICT capability. At one end of the continuum the scheme of work is rigid, the teaching tends to be mechanistic in nature and is concerned with the development of the tools within the software application under study in that lesson. On the other end of the continuum there is a method of development which involves accessing pupils' metacognitive skills using strategies such as facilitation and problem solving. School A resides at one end of the continuum, where ICT is perceived primarily as a cross-curricular tool or aid for learning other subjects, developed through a series of tasks with only superficial opportunities for evaluation or the use of higher order skills. At the other end of this continuum lies School B, where the development of ICT capability is one of a facilitation of learning by the teacher. The pupils have control over their own learning and are using their higher order metacognitive skills to define their learning and how they are going to solve the problem in hand. Lying within this continuum is school C, where ICT capability is developed using metacognitive skills, but with more scaffolding and 
teacher support. This scaffolding places it between Schools A and B on the continuum of practice, but the use of verbalisation in the formulation of the success criteria by the pupils with varying degrees of guidance by the teacher locates this school closer to School B than School A.

Similarly there appeared to be a continuum of perception, with ICT capability being perceived as the tools of specific software applications at one end and the metacognitive skills at the other.

\subsection{Implications}

The recognition of a continuum of perception of ICT capability and indeed a continuum of practice in the teaching of ICT Capability may have implications for learner progression in the subject. The variation in perception between a technical and a metacognitive ICT capability implies that learners across Wales are likely to experience different foci within ICT lessons. The case studies indicated that the teacher who perceived ICT capability as being tools-based used a more mechanistic approach to their teaching whilst those who perceive ICT capability as requiring the use of metacognitive skills tended to teach with a problem-solving approach that allows for the development of those skills. From a teaching perspective, this may mean that some pupils will continue to expect direct instruction and 'spoon-feeding' as was evident in the mechanistic approach, because this is the way they are used to being taught the subject. Furthermore, pupils taught in this way are likely to perceive ICT as simply a tool. In contrast, other pupils who have experienced a more problem-solving approach to the development of ICT capability are more likely to be able to use it as a means for solving problems and generating solutions within the work place. 11-14 year old pupils in Wales are experiencing a wide variation in the knowledge and skills as a basis for further development within examination courses. This in turn has implications for employability and the creation of a work force with sufficient ICT capability. One of the key arguments which support the need for a change in curriculum is that the present curriculum is not creating sufficient skills in problem solving and metacognition at the end of schooling in order to build an economic workforce capable of assisting Wales to compete in a digital world.

The number of contradictions arising from perceptions of ICT capability in schools, and the variation in practice across schools raise questions about the teaching of ICT in Wales, and the findings may help explain the concern that the teaching of ICT capability was no longer developing the higher order metacognitive skills required by society, which was expressed by various influential bodies during (The Royal Society, 2012). At the time of the study, this had already led to the abolition of the National Curriculum for ICT in England and speculation concerning its replacement with a curriculum based on computational thinking.

Although the curricula in the two countries had diverged since the devolution of Education to the Welsh government and the changes did not apply in Wales, the impact of these and likely further changes in England were seen to have had an impact on teachers in Wales. This is particularly true of the perceptions of ICT capability. The teacher in School A reacted to the changes as being a requirement for increased resources both at a tangible level, including equipment and an increase in teaching staff, and at an intangible level relating to specialist knowledge. In contrast to this was 
the teacher for School $\mathrm{C}$ who did not believe that there was any requirement for a change in the curriculum, but that there was a need for greater creativity in teaching the present curriculum to bring about greater development of metacognitive skills. That teacher also felt that the inclusion of aspects such as algorithms, abstraction, logic and the use of different programming languages would possibly be detrimental to the motivation of his pupils, whereas the teacher in School B wholeheartedly supported the proposed changes to the curriculum, and indeed showed enthusiasm for opportunities to teach those aspects of computer science for 11-14 year olds. Interestingly, this variation in opinion might be explained by examining the qualification backgrounds of the teachers involved which are varied with the teacher in School A having a degree in Business Information Systems, teacher in School C a degree in ICT and the teacher in School B a degree in Computer Science. There are a variety of backgrounds within ICT departments, even amongst the specialist ICT teachers, and these variations may influence their response to change depending on where their knowledge base lies. Whilst this knowledge base is linked to individuals and plays a part in defining the individual's perception, it is also likely to influence the culture of the pedagogical tools and the learning environment. It is likely that those individual teachers with a background in computer science and who exist in a community in support of computing are more likely to include computing contexts as competencies in their perception of the subject.

Since the time of the interviews, there have been proposals for change in Wales (Arthur et al. 2013) which include introducing elements of computer science to be taught alongside aspects digital competency in the new curriculum in Wales. There is also a recommendation that metacognition to support problem solving skills is explicit in this new subject area. This is crucial if this subject is to be taught on a conceptual level and not just in a mechanistic format. This study has shown that despite the inclusion and emphasis of the development of thinking skills as part of the ICT curriculum orders (ACCAC 2008), there is evidence that the subject is taught in a variety of ways and there is a possible continuum of perceptions of the subject, with a definition of ICT capability as being a tool to support learning in other subjects at one end, and a subject driven by metacognitive skills, creating appropriate applications to support problem solving at the other. If the new subject as proposed is to have an impact on the development of metacognition as part of the curriculum, then those curriculum orders are likely to require a degree of explicit explanation as this has not been obvious to all teachers in the past. In the unlikely event that the proposals are dismissed and the curriculum remains as a variation of its present form, there will still be a necessity that the subject is more adequately defined and professional development encouraged. There is a danger that if there is a wide variation in understanding of the subject, and an accompanying variation in practice then at the two different ends of that continuum the variation in the learning and hence outcomes of the activity will be pronounced.

This study is limited as it is based on the analysis of three case studies in one part of Wales and a larger study would help to increase the reliability of the conclusions and to validate the suggested continuums in perception of ICT and in pedagogical approach. However, the results of this study suggest that relying on the resolution of contradictions (Engeström 1996; 2001; Virkkunen and Kuutti 2000) to stimulate teachers' professional learning has not had a positive outcome concerning the ICT curriculum 
in Wales. It is therefore unlikely that new curriculum requirements, however clearly specified, will be satisfactorily integrated into schools' existing activity systems without externally supported professional development. Furthermore, any professional development for a new curriculum will need to take account of quite different starting points; not only do practices vary between and within schools, but teachers' personal perceptions of the very nature of the subject differ considerably from each other and from the official curriculum. Meanwhile, more research is needed concerning the factors which influence where a teacher is positioned in relation to perceptions and pedagogy, and concerning the effect on pupil attainment of these variations.

Open Access This article is distributed under the terms of the Creative Commons Attribution 4.0 International License (http://creativecommons.org/licenses/by/4.0/), which permits unrestricted use, distribution, and reproduction in any medium, provided you give appropriate credit to the original author(s) and the source, provide a link to the Creative Commons license, and indicate if changes were made.

\section{References}

ACCAC (2000). Information Technology in the National Curriculum For Wales. s.1.: ACCAC.

ACCAC (2008). Information Technology in the National Curriculum in Wales. Cardiff: Accac.

Arthur, S., Crick, T., \& Hayward, J. (2013). The ICT steering group's report to the Welsh government. Cardiff: Welsh Government.

Daniels, H., et al. (2010). Activity theory in practice. London: Routledge.

DCELLS (2008). Key stage 3 National Strategy. Cardiff: Welsh Assembly Government.

DCFS, 2002. National Strategies Secondary ICT Framework. [Online] Available at: [Accessed 20 August 2009]. DES (1985). The curriculum from 5 to 16. Curriculum matters 2. London: HMSO.

DES/WO (1990). Technology in the National Curriculum. London: DES/WO.

Engeström, Y. (1996). Interobjectivity, ideality and dialectics. Mind, Culture, and Activity, 3(4), 259-265.

Engeström, Y. (2001). Expansive learning at work: toward an activity theoretical reconceptualization. Journal of Education \& Work, 14(1), 133-156.

Engeström, Y., Meittinen, R., \& Punmaki, R.-L. (1999). Perspectives on activity theory. Cambridge: Cambridge University Press.

Gaskell, C. (2003). Capability or office fodder (pp. 22-37). SUMMER: ACITT.

Kennewell, S. (2003). Developing an ICT capability for learning. In Y. Katz (Ed.), Learning in school, home and community: ICT in early and elementary education (pp. 75-83). Boston: Kluwer Academic Press.

Kennewell, S. (2010). Analysing the impact of information technology on activity and learning. In A. McDougall, J. Murnane, A. Jones, \& N. Reynolds (Eds.), Researching IT in education: theory, practice and future directions (pp. 112-120). London: Routledge.

Kennewell, S., Parkinson, J., \& Tanner, H. (2000). Developing the ICT capable school. London: Routledge Falmer. Lankshear, C. (2010). The challenge of digital epistemologies (pp. 167-186). Communication \& Information: Education.

Markauskaite, L. (2007). Exploring the structure of trainee teachers' ICT literacy: the main components of, and relations. Educational Technology Research and Development, 55(6), 547-572.

Shulman, L. S. (1987). Knowledge and teaching: Foundation of the New Reform. Harvard Educational Review, 59(1), 1-22.

Virkkunen, J., \& Kuutti, K. (2000). Understanding organizational learning by focusing on "activity systems". Accounting, Management and Information Technologies, 10(4), 291-319.

Webb, M. E. (2002). Pedagogical Reasoning: Issues and Solutions for the Teaching and Learning of ICT in Secondary Schools. Education and Information Technologies, 7(3), 237-255.

Wilson, S. M., Shulman, L. S., \& Richart, A. E. (1987). 150 different ways of knowing: Representations of knowledge in Teaching. In: Exploring Teachers Thinking (pp. 104-124). London: s.n

Yin, R. (2009). Case study research design and methods (4th ed.). London: Sage. 